VOL. $3(1970), 81-83$.

\title{
A note on simultaneous approximation
}

\section{J. M. Mack}

We observe that good simultaneous rational approximations to given rationally dependent real numbers must satisfy the same rational dependence relationships as do the given numbers.

If $\theta_{1}, \ldots, \theta_{n} \quad(n \geq 2)$ are given real numbers, one can always find integers $p_{1}, \ldots, p_{n}, q$, with $q$ arbitrarily large, such that

$$
\left|\theta_{j}-\frac{p_{j}}{q}\right|<\frac{1}{q^{1+1 / n}}(j=1, \ldots, n) .
$$

Sets of rational fractions $\frac{p_{1}}{q}, \ldots, \frac{p_{n}}{q}$ satisfying (1) are called good simultaneous approximations to $\theta_{1}, \ldots, \theta_{n}$.

If $1, \theta_{1}, \ldots, \theta_{n}$ are linearly dependent over the rational field, we note here a simple general result connecting good simultaneous approximations to $\theta_{1}, \ldots, \theta_{n}$ with the dependence relations satisfied by these numbers. Namely, we prove the

LEMMA. Suppose the $n$ real numbers $\theta_{1}, \ldots, \theta_{n}$ satisfy $r$ linear relations

$$
\sum_{j=1}^{n} a_{i j} \theta_{j}=b_{i} \quad(i=1, \ldots, r),
$$

Received 14 April 1970. The author is indebted to Professor Mahler and Professor Szekeres for discussions on simultaneous approximation during the Tenth Summer Research Institute of the Australian Mathematical Society, held in Hobart, Tasmania, during January 1970.

81 
where the $a_{i j}$ are not all zero, and the $a_{i j}$ and $b_{i}$ are integers. Then there exists a constant $c>0$, depending on the $a_{i j}$, such that the following statement is true:

$$
\text { if } \begin{aligned}
\frac{p_{1}}{q}, \ldots, \frac{p_{n}}{q}\left(p_{j}\right. & \text { integers, } q>0 \text { an integer }) \text { satisfy } \\
& \max _{j}\left|q \theta_{j}-p_{j}\right|<c,
\end{aligned}
$$

then

$$
\sum_{j=1}^{n} a_{i j} \frac{p_{j}}{q}=b_{i} \quad(i=1, \ldots, r) .
$$

That is, simultaneous rational approximations to a set of rationally dependent real numbers either satisfy the same rational dependence relationships or else must be very bad approximations.

The proof is simple. We have, for each $i=1, \ldots, r$,

$$
\sum_{j=1}^{n} a_{i j} p_{j}=q b_{i}+\sum_{j=1}^{n} a_{i j}\left(p_{j}-q \theta_{j}\right)
$$

and so

$$
\begin{aligned}
\left|\sum_{j=1}^{n} a_{i j} p_{j}-q b_{i}\right| & \leq \sum_{j=1}^{n}\left|a_{i j}\right|\left|q^{\theta}{ }_{j}-p_{j}\right|, \\
& <c \sum_{j=1}^{n}\left|a_{i j}\right| .
\end{aligned}
$$

Thus if we choose for $c$ any positive number less than or equal to $\left(\max _{i} \sum_{j=1}^{n}\left|a_{i j}\right|\right)^{-1}$, the left hand side of the above inequality is an integer with absolute value less than 1 and so vanishes.

This result is relevant to the problem of constructing satisfactory higher dimensional generalisations of the continued fraction algorithm. We see that no such generalisation can be expected to produce good simultaneous rational approximations to sets of $n \geq 2$ arbitrarily chosen real numbers unless it is able to recognise all rational dependence 
relationships connecting the numbers. A particular instance of this observation is implicit in Perron's investigation of the generalised Jacobi algorithm (see [2], $\$ 7,8$ and [1], Kap. 14, Nr 7).

\section{References}

[1] Paul Bachmann, Die Arithmetik der quadratischen Formen. Zweite Abteizung (Teubner, Leipzig, Berlin, 1923).

[2] Oskar Perron, "Grundlagen für eine Theorie des Jacobischen Kettenbruchalgorithmus", Math. Ann. 64 (1907), 1-76.

University of Sydney,

Sydney,

New South Wales. 